\title{
NEW ANALYTIC SOLUTIONS OF THE ONE-DIMENSIONAL HEAT EQUATION FOR TEMPERATURE AND HEAT FLOW RATE BOTH PRESCRIBED AT THE SAME FIXED BOUNDARY (WITH APPLICATIONS TO THE PHASE CHANGE PROBLEM)*
}

\author{
BY \\ DAVID LANGFORD** \\ Pratt \& Whitney Aircraft, East Hartford, Connecticut
}

\begin{abstract}
New solutions of the heat equation are exhibited for the case in which both the tempcrature and heat flow rate are prescribed at a single fixed boundary. The prescribed temperature and heat flow rate may be any arbitrary infinitely differentiable functions of time. The new solutions are applicable for one-dimensional (radial) heat flow in spheres, cylinders, and slabs.

Special solutions may be obtaincd by choosing special forms for the prescribed boundary temperature and boundary heat flow rate. These special solutions include the classical solutions of the heat equation, new sequences of polynomial and quasipolynomial solutions of the heat equation, and new closed-form solutions to constantvelocity phase change problems with spherical and cylindrical symmetry.
\end{abstract}

A. Introduction. We treat the one-dimensional diffusion of heat perpendicular to the surfaces of parallel planes, coaxial cylinders, and concentric spheres. For constant density and constant thermal diffusivity, the indicated heat diffusion process is governed by the relation

$$
x^{-k} \cdot\left(x^{k} \cdot u_{x}\right)_{x}=u_{t}(x, t) .
$$

Here $u(x, t)$ is the dimensionless temperature at point $x$ at time $t$,

$x$ is the dimensionless radial position variable,

$t$ is the dimensionless time variable, and

$k=0,1,2$ for plane, cylinder, and sphere, respectively.

We solve the Cauchy problem, that is, we determine $u(x, t)$ when both the dimensionless temperature $f(t)$ and the dimensionless heat flow rate $q(t)$ are prescribed at some fixed boundary, say at $x=x_{0}$. (The dimensionless temperature at $x_{0}$ is just $u\left(x_{0}, t\right)$; the dimensionless heat flow rate at $x_{0}$ is just limit $\left._{x \rightarrow x_{0}}\left[-x^{k} \cdot u_{x}(x, t)\right]\right)$. We consider both $x_{0}=0$ and $x_{0} \neq 0$, with $k=0,1,2$.

*Received December 10, 1965; revised manuscript received May 16, 1966. This is based in part on a dissertation [4] accepted by Rensselaer Polytechnic Institute in partial fulfillment of the requirements for the degree of Doctor of Engineering Science. The author wishes to acknowledge valuable discussions with Professor Bronis R. Onuf (who suggested the thesis topic), Professor Howard W. Butler (who acted as thesis adviser) and Professor Murray R. Spiegel (who served on the Doctoral Committee). The research was supported by an educational grant from Pratt \& Whitney Aircraft Division of United Aircraft Corporation.

**Now with Drexel Institute of Technology, Philadelphia, Pa. 
We assume that $f(t)$ and $q(t)$ are analytic. Existence and uniqueness of an analytic solution are then guaranteed (in the small) (for fixed $k$ and fixed $x_{0}$ ) by a Theorem of Cauchy and Kowalewsky [2]. Further, the nonexistence of any nonanalytic solution is guaranteed by a Theorem of Holmgren [2].

'The analytic solutions given here correspond to physical reality because [2] they depend continuously on the prescribed data. The use of these solutions is demonstrated for several cases of physical interest. We now outline the procedure used to obtain the solutions.

For $x_{0}=0$, we may assume that $u(x, t)=\sum_{m, n}\left(a_{m n}+b_{m n} \cdot \ln _{e} x\right) x^{m} t^{n}$, substitute into the heat diffusion equation, and obtain recursion relations for the constants $a_{m n}$ and $b_{m n}$. The recursion relations may be used to decompose $u(x, t)$ into series of polynomial and quasi-polynomial solutions of the heat equation. These series may be summed to obtain the final solutions to the Cauchy problem. The final solutions are presented with the derivation being omitted; the elements of the series are exhibited as special cases of the final solutions.

For $x_{0} \neq 0$ : the solution $u^{p}(x, t)$ for the planar case is a linear transformation of the solution to the Cauchy problem for $x_{0}=0$; the solution $u^{s}(x, t)$ for the spherical case is readily obtained from $u^{p}(x, t)$ when it is noted that $\left(x \cdot u^{s}\right)$ must satisfy the planar heat equation; the solution $u^{c}(x, t)$ for the cylindrical case is obtained in the Appendix. We now exhibit the solutions and illustrate their use.

B. The fixed boundary at the origin of coordinates. Consider first the case $x_{0}=0$. Let $f(t)$ and $q(t)$ be any infinitely differentiable functions. Define

$$
f^{n}(t) \equiv\left(d^{n} / d t^{n}\right) f(t) \quad \text { and } \quad q^{n}(t) \equiv\left(d^{n} / d t^{n}\right) q(t) .
$$

Then it may be verified by differentiation that the following are formal solutions of the heat equation:

plane,

$$
u(x, t)=\sum_{n=0}^{\infty}\left[\frac{f^{n}(t) \cdot x^{2 n}}{(2 n) !}-\frac{q^{n}(t) \cdot x^{2 n+1}}{(2 n+1) !}\right]
$$

sphere, $\quad u(x, t)=\sum_{n=0}^{\infty}\left[\frac{f^{n}(t) \cdot x^{2 n}}{(2 n+1) !}+\frac{q^{n}(t) \cdot x^{2 n-1}}{(2 n) !}\right]$;

cylinder, $\quad u(x, t)=\sum_{n=0}^{\infty} \frac{(x / 2)^{2 n}}{n ! \cdot n !} \cdot\left[f^{n}(t)-q^{n}(t) \cdot\left\{\ln _{\bullet} x-\frac{1}{1}-\frac{1}{2}-\cdots-\frac{1}{n}\right\}\right]$,

where the factor in braces is just $\left\{\ln _{0} x\right\}$ for $n=0$. These solutions have the property that $\operatorname{limit}_{x \rightarrow 0}\left[-x^{k} \cdot u_{x}(x, t)\right]=q(t)$; this makes it possible to prescribe the heat flow rate at $x_{0}$ as an arbitrary infinitely differentiable function $q(t)$. For the planar case, the solution $2 \mathrm{a}$ has the property that $u(0, t)=f(t)$; this makes it possible to prescribe the temperature at $x_{0}$ as an arbitrary infinitely differentiable function $f(t)$. For the spherical and cylindrical cases, if $q(t) \neq 0$, then it is physically necessary that $u(0, t)=\infty$, as required by Eqs. $2 \mathrm{~b}$, c; if $q(t)=0$, then $u(0, t)=f(t) .{ }^{*}$

The above solutions are general solutions of the heat equation in that they may be specialized to yield other solutions of the heat equation. For example, the classical

*The solution 2a was given by Stefan [8] in 1890; he used it to compute the thermal conductivity of ice. The solutions $2 \mathrm{~b}, \mathrm{c}$ have not been previously published, though several of the leading terms of the solution 2c were given by Pekeris and Slichter [6] in 1939. 
Fourier series and Bessel series solutions of the heat equation may be obtained from Eqs. 2a, b, c by choosing

$$
f(t)=\sum_{m=0}^{\infty} A_{m} \cdot \exp \left(-b_{m}^{2} t\right) \text { and } q(t)=\sum_{m=0}^{\infty} B_{m} \cdot \exp \left(-b_{m}^{2} t\right) .
$$

New solutions of the heat equation may also be obtained from Eq. 2. For example, if

$$
f(t)=\sum_{n=0}^{\infty}\left(f_{n} t^{n} / n !\right) \text { and } q(t)=\sum_{n=0}^{\infty}\left(q_{n} t^{n} / n !\right),
$$

then direct substitution into Eqs. 2a, b, c yields

$$
\begin{array}{ll}
\text { plane, } & u(x, t)=\sum_{n=0}^{\infty}\left[f_{n} \cdot P^{2 n}(x, t)-q_{n} \cdot P^{2 n+1}(x, t)\right] ; \\
\text { sphere, } & u(x, t)=\sum_{n=0}^{\infty}\left[f_{n} \cdot S^{2 n}(x, t)+q_{n} \cdot S^{2 n-1}(x, t)\right] ; \\
\text { cylinder, } & u(x, t)=\sum_{n=0}^{\infty}\left[f_{n} \cdot C^{2 n}(x, t)-q_{n} \cdot E^{2 n}(x, t)\right] .
\end{array}
$$

The $P^{m}(x, t), S^{m}(x, t)$, and $C^{m}(x, t)$ appearing in these relations are polynomial solutions of the planar, spherical, and cylindrical heat equations, respectively, with

$$
\begin{gathered}
P^{2 n}(x, t) \equiv x \cdot S^{2 n-1}(x, t) \equiv t^{n} / n !+t^{n-1} \cdot x^{2} /(n-1) ! 2 ! \\
\quad+t^{n-2} \cdot x^{4} /(n-2) ! 4 !+\cdots+t^{0} \cdot x^{2 n} / 0 !(2 n) ! \\
\begin{aligned}
P^{2 n+1}(x, t) \equiv x \cdot S^{2 n}(x, t)_{-} \equiv t^{n} \cdot x^{1} / n ! 1 !+t^{n-1} \cdot x^{3} /(n-1) ! 3 ! \\
+
\end{aligned} \\
t^{n-2} \cdot x^{5} /(n-2) ! 5 !+\cdots+t^{0} \cdot x^{2 n+1} / 0 !(2 n+1) ! \\
C^{2 n}(x, t) \equiv t^{n} / n !+x^{2} \cdot t^{n-1} / 2^{2} \cdot(n-1) !+x^{4} \cdot t^{n-2} / 4^{2} 2^{2} \cdot(n-2) ! \\
+\cdots+x^{2 n} \cdot t^{0} /(2 n)^{2}(2 n-2)^{2} \cdots 4^{2} 2^{2} \cdot 0 ! .
\end{gathered}
$$

The $E^{2 n}(x, t)$ are quasi-polynomial solutions of the cylindrical heat equation with

$$
\begin{aligned}
E^{2 n}(x, t) \equiv t^{n} & \left(\ln _{\bullet} x\right) / n !+x^{2} \cdot t^{n-1}\left(\ln _{e} x-1\right) / 2^{2} \cdot(n-1) !+\cdots \\
& \quad+x^{2 n} \cdot t^{0}\left(\ln _{\bullet} x-1-\frac{1}{2}-\cdots-\frac{1}{n}\right) /(2 n)^{2}(2 n-2)^{2} \cdots 4^{2} 2^{2} \cdot 0 ! .
\end{aligned}
$$

The $S^{m}(x, t), C^{2 n}(x, t)$, and $E^{2 n}(x, t)$, are new solutions. ${ }^{*}$ Further,

$$
x^{-k} \cdot\left(x^{k} \cdot V_{x}^{m}\right)_{x}=V_{t}^{m}(x, t)=V^{m-2}(x, t) \text { for } V^{m}=P^{m}, S^{m}, C^{2 m}, E^{2 m}
$$

C. The fixed boundary not at the origin of coordinates. There exist solutions $u(x, t)$ of the heat equation such that $u\left(x_{0}, t\right)=f(t)$ and such that $\operatorname{limit}_{x \rightarrow x_{0}}\left[-x^{k} \cdot u_{x}(x, t)\right]=q(t)$, where $x_{0}>0$ and where $f(t)$ and $q(t)$ are arbitrarily prescribed infinitely differentiable functions. The solution for the planar case is a lincar transformation of the solution 2a; the solutions for the spherical and cylindrical cases have not bcen previously published.

${ }^{*}$ The planar heat polynomials, through $P^{6}(x, t)$, were given by Carslaw and Jaeger [1] in 1959, though these authors used a normalization different from that used here. The planar heat polynomials, of the type used by Carslaw and Jaeger, were treated extensively by Rosenbloom and Widder [7] in 1959 and subsequently by Widder $[9,10]$. 
The notation of Eqs. 1 may be used to write the solutions for the three cases as follows:

plane,

$$
u(x, t)=\sum_{n=0}^{\infty}\left[f^{n}(t) \frac{\left(x-x_{n}\right)^{2 n}}{(2 n) !}-q^{n}(t) \frac{\left(x-x_{n}\right)^{2 n+1}}{(2 n+1) !}\right]
$$

sphere, $\quad u(x, t)=\sum_{n=1}^{\infty}\left[f^{n}(t) \frac{\left(x-x_{n}\right)^{2 n}}{(2 n) !} \cdot \frac{x+2 n x_{n}}{(2 n+1) x}-q^{n}(t) \frac{\left(x-x_{n}\right)^{2 n+1}}{(2 n+1) ! \cdot x x_{0}}\right]$;

cylinder, $\quad u(x, t)=\sum_{n=0}^{\infty}\left[f^{n}(t) \cdot c^{n}\left(x^{2} / 4\right)-\frac{1}{2} \cdot q^{n}(t) \cdot e^{n}\left(x^{2} / 4\right)\right]$.

The functions $c^{n}\left(x^{2} / 4\right)$ and $e^{n}\left(x^{2} / 4\right)$ are defined in the appendix.

The solutions 7 may be used, as in Sec. B, to obtain classical-type solutions to the heat equation and also to obtain new polynomial and quasi-polynomial solutions to the heat equation. The solutions 2 and 7 may also be used to obtain new solutions to phase change problems. This will now be demonstrated.

D. Applications to the phase change problem. The addition of heat to a physical system can cause motion of a phase boundary, say of an ice-water interface. We assume that phase change occurs at a constant temperature $u=u^{*}$. As a consequence, for a symmetric one-dimensional system, the location $x=y(t)$ of the moving phase boundary is defined by the implicit relation

$$
u(x, t)=u^{*}=\text { constant at } x=y(t) .
$$

The general solutions given in sect.ons $\mathrm{B}$ and $\mathrm{C}$ will be used to obtain closed form solutions to phase change problems in which the jsothermal phase boundary is assumed to move at constant velocity $y_{1}$, with the temperature being zero at $x=y(t)=y_{0}+y_{1} t$.

Consider the molting of a slab or sphere which is initially at the phase change temperature $u=0$. For $t>0$ there is assumed to be a heat input at $x=y(0)$. This heat input causes melting and results in the creation of a nonisothermal liquid extending from $y(0)$ to the moving phase boundary at $x=y(t)$; the material adjacent to $y(t)$ will be in the solid state with $u=0$. The temperature $u(x, t)$ in the liquid region maly be determined from knowledge of the temperature $f(t)$ and heat flow rate $q(t)$ at $x=x_{0}=y_{0}=y(0)$. For the slab case $(k=0)$ and constant velocity melting it has been shown elsewhere [4] that

and that

$$
f(t)=\left\{\exp \left[y_{1}\left(y-x_{11}\right)\right]-1\right\}
$$

$$
q(t)=y_{1} \cdot \exp \left[y_{1}\left(y-x_{0}\right)\right]
$$

Substitution of equations $9 \mathrm{a}, \mathrm{b}$ into the general solution $7 \mathrm{a}$ yiclds

$$
u(x, t)=\left\{\exp \left[y_{1}(y-x)\right]-1\right\} ; \quad y=y_{0}+y_{1} t .^{*}
$$

For the spherical case $(k=2)$ and constant velocity melting it has been shown elsewhere [4] that

$$
f(t)=\left(1-2 / y_{0} y_{1}\right) \cdot\left(\exp \left[y_{1}^{2} t\right]-1\right)+\left(2 y_{1} t / y_{0}\right) \cdot \exp \left[y_{1}^{2} t\right],
$$

and that

$$
q(t)=\left(2 / y_{1}\right)+\left(2 / y_{1}\right) \cdot\left[\left(y_{0}^{2} y_{1}^{2} / 2\right)-1+\left(y_{0} y_{1}+1\right) \cdot y_{1}^{2} t\right] \cdot \exp \left[y_{1}^{2} t\right]
$$

\footnotetext{
*The solution $9 \mathrm{c}$ was given by Stefan [8] in 1890 .
} 
Substitution of equations $10 \mathrm{a}, \mathrm{b}$ into the general solution $7 \mathrm{~b}$ yields

$$
\begin{array}{r}
u(x, t)=\left(1-2 / x y_{1}\right)\left[\exp \left[y_{1}(y-x)\right]-1\right]-2(1-y / x) \cdot \exp \left[y_{1}(y-x)\right] \\
y=y_{0}+y_{1} t .
\end{array}
$$

This solution is new. It has recently been given elsewhere [3] in dimensional form, but without derivation.

The solution $2 \mathrm{c}$ of the cylindrical heat equation can be used directly to obtain the solution to a constant velocity phase change problem. To demonstrate this, note that the solution $2 \mathrm{c}$ will satisfy the boundary condition 8 if

$$
f^{n}(t)=q^{n}(t) \cdot\left[\ln _{e} y(t)-1-\frac{1}{2}-\cdots \frac{1}{n}\right]
$$

If this relation is valid, then the solution $2 \mathrm{c}$ may be written

$$
u(x, t)=\sum_{n=0}^{\infty}(x / 2)^{2 n} \cdot(1 / n !)^{2} \cdot q^{n}(t) \cdot \ln _{e}(y / x) .
$$

The $q^{n}(t)$ must satisfy Eq. 1, but for the function $11 \mathrm{~b}$ to satisfy the cylindrical heat equation it is also necessary that

$$
q_{\iota}^{n}(t) / q^{n}(t)=-(n+1) \cdot y_{\iota}(t) / y(t) ; \quad n=0,1,2, \cdots .
$$

Direct integration of this relation yields

$$
q^{n}(t) \cdot y^{n+1}(t)=q^{n}(0) \cdot y^{n+1}(0)=\text { constant. }
$$

For $n=0$, equation $11 \mathrm{~d}$ yields

$$
q^{0}(t) \cdot y(t)=q^{0}(0) \cdot y(0)=\text { constant. }
$$

Now $q^{1}(t)=q_{t}^{0}(t)$ by equation 1 so $q_{t}^{1}(t) / q^{1}(t)=q_{t t}^{0}(t) / q_{t}^{0}(t)$. Derivatives of equation $11 \mathrm{e}$ may be substituted into the right side of this relation to show that

$$
q_{t}^{1}(t) / q^{1}(t)=y_{\iota t}(t) / y_{\imath}(t)-2 \cdot y_{\imath}(t) / y(t) .
$$

For the result $11 \mathrm{f}$ to be compatible with the condition $11 \mathrm{c}$ it is necessary that $y_{\mathfrak{t}}(t)=0$. From this it follows that

$$
y(t)=y_{0}+y_{1} t, \quad \text { with } y_{0}=y(0) \text { and } y_{1}=y_{\iota}(0) .
$$

Eq. 11e can now be used with the result $11 \mathrm{~g}$ and the definition 1 to show that

$$
q^{n}(t)=-\left(-y_{1} / y\right)^{n+1} \cdot\left(q_{0} y_{0} / y_{1}\right) \cdot(n !), \quad q_{0} \equiv q(0) .
$$

Substitution of the result $11 \mathrm{~h}$ into Eq. $11 \mathrm{~b}$ yields the following solution of the cylindrical heat equation:

$u(x, t)=\left(v^{*} / y\right) \cdot \ln _{e}(y / x) \cdot \exp \left(-x^{2} y_{1} / 4 y\right), \quad v^{*}=$ constant $=q_{0} y_{0} ;$

or

$u(x, t)=\frac{w^{*}(x / y)}{\left(x y_{1} / 4\right)} \cdot \ln _{\bullet}\left(\frac{x}{y}\right) \cdot \exp -\left[\frac{x}{y}\left(x y_{1} / 4\right)\right]$,

$$
w^{*}=\text { constant }=-q_{0}\left(y_{0} y_{1} / 4\right) .
$$


This result has not been previously published. It may be interpreted as the solution to a constant velocity phase change problem because it satisfies the boundary condition 8 .

E. Closure. Sections B and C contain solutions of the heat equation which permit the temperature and/or the heat flow rate at a fixed boundary to be arbitrarily prescribed infinitely differentiable functions. These solutions have been used to obtain the classical solutions of the heat equation, sequences of polynomial and quasi-polynomial solutions of the heat equation, and new closed form solutions to constant velocity phase change problems.

The closed form solutions to the phase change problem which have becn developed here can be written in a common form. If it is assumcd that there exist other solutions to the phase change problem which have the same form, then it is possible to develop additional general solutions of the heat equation which may be specialized to yield new closed form solutions to various phase change problcms. This development is given in a companion paper [5].

F. Appendix. We will construct a function $u(x, t)$ such that

$$
\begin{aligned}
\left(x \cdot u_{x}\right)_{x} & =x \cdot u_{t}(x, t), \\
u\left(x_{0}, t\right) & =f(t), \\
-x_{0} \cdot u_{x}\left(x_{0}, t\right) & =q(t) .
\end{aligned}
$$

Here $f(t)$ and $q(t)$ are arbitrary infinitcly differentiable functions and $x_{0}$ is a positive constant. To simplify the construction, define

$$
\begin{gathered}
z \equiv x^{2} / 4, \quad z_{0} \equiv x_{0}^{2} / 4 ; \\
v(z, t) \equiv u(x, t) .
\end{gathered}
$$

The cylindrical heat equation 1 and the boundary conditions 2, 3 can now be written

$$
\begin{aligned}
\left(z \cdot v_{z}\right)_{z} & =v_{t}(z, t) ; \\
v\left(z_{0}, t\right) & =u\left(x_{0}, t\right)=f(t) ; \\
-z_{0} \cdot v_{z}\left(z_{0}, t\right) & =-\frac{1}{2} \cdot x_{0} \cdot u_{x}\left(x_{0}, t\right)=\frac{1}{2} \cdot q(t) .
\end{aligned}
$$

We begin the construction by assuming that

$$
v(z, t)=\sum_{n=0}^{\infty}\left[f^{n}(t) \cdot c^{n}(z)-\frac{1}{2} \cdot q^{n}(t) \cdot e^{n}(z)\right] .
$$

This form is assumed because it is the form satisfied by the previously given solutions of the planar and spherical heat cquation. Here $c^{n}(z)$ and $e^{n}(z)$ are unknown sequences of functions and

$$
\begin{aligned}
f^{n}(t) & \equiv\left(d^{n} / d t^{n}\right) f(t) ; \\
q^{n}(t) & =\left(d^{n} / d t^{n}\right) q(t) .
\end{aligned}
$$

The form 9 will satisfy the transformed heat Eq. 6 if

$$
\begin{array}{ll}
\left(z \cdot c_{z}^{n}\right)_{z}=c^{n-1}(z), & \left(z \cdot c_{z}^{0}\right)_{z}=0 ; \\
\left(z \cdot e_{z}^{n}\right)_{z}=e^{n-1}(z), & \left(z \cdot e_{z}^{0}\right)_{z}=0 .
\end{array}
$$


The form 9 will also satisfy the transformed boundary conditions 7, 8 if

$$
\begin{gathered}
c^{n}\left(z_{0}\right)=\delta_{n 0}, \quad c_{z}^{n}\left(z_{0}\right)=0 ; \\
e^{n}\left(z_{0}\right)=0, \quad z_{0} \cdot e_{z}^{n}\left(z_{0}\right)=\delta_{n 0}, \text { the Kronecker delta. }
\end{gathered}
$$

The $c^{n}(z)$ and $e^{n}(z)$ can be uniquely determined by integrating the defining relations 12 and 13 subject to the conditions 14 and 15. Several of the $c^{n}(z)$ and $e^{n}(z)$ follow.

$c^{0}(z)=1$,

$c^{1}(z)=\left(z-z_{0}\right)-z_{0} L$, with $L \equiv \ln _{\mathrm{e}}\left(z / z_{0}\right)$,

$c^{2}(z)=\left(z^{2}-z_{0}^{2}\right) / 4+z_{0}\left(z-z_{0}\right)-z_{0}\left(z+z_{0} / 2\right) L$,

$c^{3}(z)=\left(z^{3}-z_{0}^{3}\right) / 36+z_{0}\left(z^{2}-z_{0}^{2}\right) / 2-z_{0}^{2}\left(z-z_{0}\right) / 4-z_{0}\left(z^{2} / 4+z_{0} z / 2+z_{0}^{2} / 12\right) L ;$

$e^{0}(z)=L \equiv \ln _{e}\left(z / z_{0}\right)=\ln _{e}\left(x^{2} / x_{0}^{2}\right)$,

$e^{1}(z)=z\left(L-\frac{2}{1}\right)+z_{0}\left(L+\frac{2}{1}\right)$,

$e^{2}(z)=\left(L-\frac{2}{1}-\frac{2}{2}\right) \frac{z^{2}}{2^{2}}+L z z_{0}+\left(L+\frac{2}{1}+\frac{2}{2}\right) \frac{z_{0}^{2}}{2^{2}}$,

$e^{3}(z)=\left(L-\frac{2}{1}-\frac{2}{2}-\frac{2}{3}\right) \frac{z^{3}}{3 ! 3 !}+\left(L-\frac{2}{2}\right) z^{2} z_{0} / 2^{2}$

$$
+\left(L+\frac{2}{1}+\frac{2}{2}+\frac{2}{3}\right) \frac{z_{0}^{3}}{3 ! 3 !}+\left(L+\frac{2}{2}\right) z_{0}^{2} z / 2^{2} .
$$

The asymmetric form of the $c^{n}(z)$ makes it difficult to obtain a simple functional expression for $c^{n}(z)$ for arbitrary $n$. It is however possible to define $c^{n}(z)$ as a linear combination of $z^{n}$ and of all $c^{m}(z)$ and $e^{m}(z)$ for which $0 \leqq m<n$. Similarly for the $e^{n}(z)$. This will be demonstrated.

Let $n$ be a positive integer and, for later use, define

$$
\phi^{n}(z) \equiv\left(z^{n}-z_{0}^{n}\right) \text { and } \psi^{n}(z) \equiv z^{n} \cdot \ln \bullet\left(z / z_{0}\right) .
$$

The functions $\phi^{n}(z)$ and $\psi^{n}(z)$ have the property that

$$
\begin{aligned}
& \int_{z_{0}}^{z}\left(d z^{\prime} / z^{\prime}\right) \int_{z_{0}}^{z^{\prime}} \phi^{n}\left(z^{\prime \prime}\right) d z^{\prime \prime}=\frac{\phi^{n+1}(z)}{(n+1)^{2}}-z_{0}^{n}\left(c^{1}+\frac{z_{0} e^{0}}{n+1}\right) ; \\
& \int_{s_{0}}^{z}\left(d z^{\prime} / z^{\prime}\right) \int_{z_{0}}^{z^{\prime}} \psi^{n}\left(z^{\prime \prime}\right) d z^{\prime \prime}=\frac{\psi^{n+1}(z)}{(n+1)^{2}}-2 \frac{\phi^{n+1}(z)}{(n+1)^{3}}+\frac{z_{0}^{n+1} e^{n}(z)}{(n+1)^{2}} .
\end{aligned}
$$

We begin the demonstration by using definition $18 \mathrm{a}$ and result $17 \mathrm{a}$ to rewrite $16 \mathrm{~b}$ and $17 \mathrm{~b}$ thus:

$$
\begin{aligned}
c^{1}+z_{0} e^{0} & =\phi^{1} \\
e^{1}+2 \phi^{1} & =\psi^{1}+z_{0} e^{0} .
\end{aligned}
$$

We now use the defining relations 12 and 13 to rewrite 19 and 20 in the form

$$
\begin{aligned}
\left(z c_{z}^{2}\right)_{z}+z_{0}\left(z e_{2}^{1}\right)_{z} & =\phi^{1} ; \\
\left(z e_{z}^{2}\right)_{z}+2 \phi^{1} & =\psi^{1}+z_{11}\left(z e_{z}^{1}\right)_{z} .
\end{aligned}
$$


Expressions for $c^{2}(z)$ and $e^{2}(z)$ may be obtained by integrating equations 21 and 22 and making use of the boundary conditions 14 and 15. The right sides of the integrated equations may be evaluated by use of equations $18 \mathrm{~b}$, c. The resulting equations can then be used with relations 12 and 13 to begin a similar procedure for the calculation of $c^{3}(z)$ and $e^{3}(z)$. It can be shown by induction that the following are the general expressions for $c^{n}(z)$ and $e^{n}(z)$ for $n>0$.

$$
\begin{aligned}
\frac{z_{0}^{0}}{(0 !)^{2}}\left[c^{n}+\frac{z_{n} e^{n-1}}{1}\right] & +\frac{z_{n}^{1}}{(1 !)^{2}}\left[c^{n-1}+\frac{z_{n} e^{n-2}}{2}\right]+\frac{z_{n}^{2}}{(2 !)^{2}}\left[c^{n-2}+\frac{z_{n} e^{n-3}}{3}\right]+\cdots \\
& +\frac{z_{0}^{n-2}}{((n-2) !)^{2}}\left[c^{2}+\frac{z_{n} e^{1}}{n-1}\right]+\frac{z_{0}^{n-1}}{((n-1) !)^{2}}\left[c^{1}+\frac{z_{n} e^{0}}{n}\right]=\frac{\phi^{n}(z)}{(n !)^{2}} ; \\
e^{n}(z)+\frac{\phi^{n}(z)}{(n !)^{2}}\left[\frac{2}{1}+\right. & \left.\frac{2}{2}+\frac{2}{3}+\cdots+\frac{2}{n}\right]=\frac{\psi^{n}(z)}{(n !)^{2}} \\
& +\left\{\frac{z_{n}^{n} e^{0}}{(n !)^{2}}+\frac{z_{0}^{n-1} e^{1}}{((n-1) !)^{2}}+\cdots+\frac{z_{n}^{2} e^{n-2}}{(2 !)^{2}}+\frac{z_{n}^{1} e^{n-1}}{(1 !)^{2}}\right\} \\
& +\frac{z_{0}^{n-1}}{((n-1) !)^{2}}\left[\frac{2}{1}+\frac{2}{2}+\frac{2}{3}+\cdots+\frac{2}{n-1}\right]\left(c^{1}+\frac{z_{n} e^{0}}{n}\right) \\
& +\frac{z_{0}^{n-2}}{((n-2) !)^{2}}\left[\frac{2}{1}+\frac{2}{2}+\frac{2}{3}+\cdots+\frac{2}{n-2}\right]\left(c^{2}+\frac{z_{0} e^{1}}{n-1}\right)+\cdots \\
& +\frac{z_{0}^{2}}{(2 !)^{2}}\left[\frac{2}{1}+\frac{2}{2}\right]\left(c^{n-2}+\frac{z_{n} e^{n-3}}{3}\right)+\frac{z_{0}^{1}}{(1 !)^{2}}\left[\frac{2}{1}\right]\left(c^{n-1}+\frac{z_{n} e^{n-2}}{2}\right)
\end{aligned}
$$

These relations may be used to compute $c^{n}(z)$ and $e^{n}(z)$ for $n>0$. The use of the $c^{n}(z)$ and $e^{n}(z)$ has been illustrated elsewhere [4].

\section{ReFerences}

1. H. S. Carslaw and J. C. Jaeger, Conduction of heat in solids, 2nd Ed., Oxford Univ. Press, London, 1959

2. R. Courant and D. Hilbert, Methods of mathematical physics, Vol. 2, Partial Differential Equations, pp. 39-54, 227, 237-9. Interscience, New York, 1962

3. David Langford, $A$ closed form solution for the constant velocity solidification of spheres initially at the fusion temperature, Br. J. Applied Physics, 17(2), 286 (1966)

4. David Langford, Stefan's melting problem, Doctoral Dissertation, Rensselaer Polytechnic Institute, Troy, N. Y., 1965

5. David Langford, Pseudo-similarity solutions of the one-dimensional diffusion equation with applications to the phase change problem, Quart. Appl. Math., 1967 (to appear)

6. C. L. Pekeris and L. B. Slichter, Problems of ice formation, J. Applied Physics, 10(2), 135-137 (1939)

7. P. C. Rosenbloom and D. V. Widder, Expansions in terms of heat polynomials and associated functions, Trans. Amer. Math. Soc. 92, 220-266 (1959)

8. J. Stefan, On the theory of ice formation, especially on ice formation in polar seas (Utber die Theorie der Eisbildung, insbesondere uber die Eisbildung im Polarmeere). Sitzungsberichte der Kaiserlichen Akademie Wiss. Wien., Math.-naturwiss. Kl. 98(2a), 965-973 (1890). (In German)

9. D. V. Widder, Analytic solutions of the heat equation, Duke Math. J. 29(4), 497-504 (1962)

10. D. V. Widder, Series expansions of solutions of the heat equation in $n$ dimensions. Ann. Mat. Pura Appl. IV-55, 389-410 (1961) (In English) 